A Qualitative Investigation into the Cultural Master Narrative for Overcoming Trauma and Adversity in the United Kingdom

\author{
Laura E. R. Blackie \\ School of Psychology \\ University of Nottingham \\ Jade E. V. Colgan \\ School of Psychology \\ University of Nottingham \\ Stephanie McDonald \\ School of Psychology \\ University of Nottingham \\ Kate C. McLean \\ Department of Psychology \\ Western Washington University
}

Accepted for publication in Qualitative Psychology. (C) 2020, American Psychological Association. This paper is not the copy of record and may not exactly replicate the final, authoritative version of the article. Please do not copy or cite without authors' permission. The final article will be available, upon publication, via its DOI: 10.1037/qup0000163

Author Note: Laura E. R. Blackie, School of Psychology, University of Nottingham, UK; Jade E. V. Colgan School of Psychology, University of Nottingham, UK; Stephanie McDonald, School of Psychology, University of Nottingham, UK; Kate K. C. McLean, Department of Psychology, Western Washington University, USA. 
Acknowledgments: We would like to thank Katie Turner for her assistance in providing the frequency counts for the main themes along with the two anonymous reviewers and action editor for their helpful comments on an earlier version of this manuscript. This publication was made possible through the support of grant \#60699 from the John Templeton Foundation. The opinions expressed in this publication are those of the authors and do not necessarily reflect the views of the John Templeton Foundation.

Correspondence concerning this article should be addressed to Laura E. R. Blackie, School of Psychology, University of Nottingham, University Park Campus, Nottingham, Nottinghamshire, NG7 2RD, UK. Email: laura.blackie@nottingham.ac.uk 
A Qualitative Investigation into the Cultural Master Narrative for Overcoming Trauma and Adversity in the United Kingdom

Research in the field of narrative psychology has found that redemption - a narrative sequence in which people recount emotionally negative experiences as having positive endings - is a useful mechanism for coping with adversity. Redemption has been viewed as a cultural master narrative in North America, providing individuals with a socially valued script for narrating challenging life experiences. Presently little research has examined the presence and function of the redemption narrative outside of North American contexts. The aim of this qualitative study, therefore, was to identify themes in UK individuals' narratives of trauma and adversity to gain insight into the content of the master narrative for meaning-making in the UK. Sixty-Five participants (57 females, Mage $=21.97, S D=7.24$ ) with little to no experience of lifetime adversity were recruited into an online survey. Participants answered open-ended questions adopting the perspective of a survivor from a selected UK national tragedy, focusing on how they felt survivors could recover from trauma. We identified two themes in our thematic analysis that were relevant to recovery: recuperation and redemption. Recuperation was most commonly reported, it was described as a gradual lessening of symptoms over time and the ability to cope with the lasting emotional and physical scars. Our findings suggest that redemption is not necessarily the dominant cultural script in the UK for guiding recovery in the aftermath of trauma.

Key words: Master Narrative, Meaning-Making, Trauma, Recovery, and Redemption 
Narrative identity is developed through a process of re-constructing memories about life experiences and recounting them in stories (Singer, 2004). The function of life stories is to construct a coherent personal identity that connects our past, present and anticipated future selves in a manner that infuses our experiences with a sense of unity across time, meaning, and purpose in life (McAdams, 1996). Unsurprisingly, integration of positive experiences into narrative identity is generally less effortful, because at least in Western societies, people tend to assume good things happen to good people (Lerner, 1980). In contrast, people find reconciling the impact of negative experiences into their identity to be far more challenging (Blackie, Cozzolino, \& Sedikides, 2016). At the most basic level, negative experiences are likely to disrupt our expectations, requiring some effortful work to understand why an experience happened and what it means to us. Consequentially, research has found that people are more likely to find meaning from events with some degree of conflict (Thorne, McLean, \& Lawrence, 2004; McLean \& Thorne, 2003). At a more extreme level, some have argued that traumatic life experiences have the potential to shatter a person's beliefs about the benevolence and meaningfulness of the world (Janoff-Bulman, 2010), leaving the individual searching to reconcile their present with their pre-trauma identity (Park, 2010).

Although the search for meaning is a deeply personal process, it does not occur in a social vacuum. The re-construction of a person's narrative identity is shaped by the cultural scripts within their society that detail the most adaptive and socially-acceptable means to draw meaning from challenging life experiences (Adler \& Poulin, 2009; Hammack, 2008; McLean \& Syed, 2016). These cultural scripts, known as 'master narratives' are shared stories within specific cultures that shape beliefs, values, and behaviour in specific circumstances. Master narratives are part of the structure of society, and as such provide guidance to members of that society about how to live a good and meaningful life (McLean \& Syed, 2016). McAdams (2006) has argued that the redemption narrative, where people narrate emotionally negative experiences as having positive endings, is a master narrative that is particularly salient for American individuals. Indeed, 
research has found that use of this narrative script is expected in narrating difficult experiences (e.g., Thorne \& McLean, 2003), and is also consistently linked to well-being in North America (Adler et al., 2015; Adler, Lodi-Smith, Philippe, \& Houle, 2016; Dunlop \& Tracy, 2013). Presently little research has examined the presence and function of the redemption narrative outside of North America, and it is unknown whether redemption is a master narrative for guiding meaning-making processes about negative events in other Western countries. The aim of this qualitative study, therefore, is to explore the narrative themes individuals in the UK use when narrating stories of trauma and adversity to gain insight into the content of the master narrative for meaning-making in the UK.

Redemption as a North American Master Narrative:

Research in North America shows that individuals who narrate their life stories using redemption report lower levels of distress, higher levels of well-being and greater investment in prosocial causes (Adler et al., 2015; McAdams \& Guo, 2015). However, redemption is not only a salient script that people in North America use to narrate challenging experiences, it functions as a coping mechanism, helping people to find meaning and reconcile difficult experiences (Breen \& McLean, 2017). Initially, the relationship between redemption and well-being was evidenced in correlational studies, but it was found among youth, middle-aged and older adult populations (McLean \& Breen, 2009; McLean \& Lilgendahl, 2008; McAdams, Reynolds, Lewis, Patten, \& Bowman, 2001). More recently however, longitudinal research has confirmed redemption is a highly adaptive narrative sequence for promoting adjustment and well-being after a diverse range of challenging life experiences. Slotter and Ward (2015) for example, found using the redemption narrative helped people manage distress following the resolution of their romantic relationships. Alder et al. (2015) found that narrating both low points and health challenges using redemption predicted improvements in mental health over a 4-year period, and Dunlop and Tracy (2013) 
found that use of the redemption narrative predicted health benefits in a sample of individuals recovering from alcoholism.

However, of particular relevance to the current study, there is evidence supporting the value of the redemptive narrative in helping people cope with and reconcile the impact of national tragedies of societal importance. Adler and Poulin (2009) examined the relationship between narrative style and well-being in a nationally representative sample in the USA 2-months after the 9/11 terrorist attacks. In these accounts, participants wrote about their experiences and efforts to make sense of this national tragedy. Critically, only $6 \%$ of this sample were personally affected, but the 9/11 attacks were interpreted as an attack on the political and cultural ideals of American life, and as such participants' well-being was higher when their narrative accounts about this public event were high in closure and national redemption, where they described how the political and cultural character of the country was strengthened in the wake of adversity. In contrast, individuals whose accounts were high in national contamination, where they narrated that the political and cultural character of the country was irrevocably damaged by the $9 / 11$ attacks, reported the highest levels of psychological distress.

In summary, these studies demonstrate that the redemption narrative is a salient cultural script in North America that promotes adjustment and well-being in the wake of both personal and shared experiences of adversity. McAdams (2013) has claimed the origin of the redemption narrative can be historically traced to the pursuit of religious freedom by the Puritans and goal of a new self-made life of opportunity by the individuals and families fleeing Europe for the New World. Redemption is, therefore, ingrained into the national ethos of the American Dream that governs the USA, as well as in the common interpretations (stories) of American history (McAdams, 2006). Yet if the "redemptive self is a quintessentially American identity" (McAdams, 2013, p. 268), then it is plausible to assume that this narrative will not necessarily guide meaning-making of challenging life experiences in other Western societies. 
The aim of this study was to explore the content of the master narrative in the UK that guides the storying process for meaning-making for highly challenging and traumatic life experiences, which to the present authors' knowledge has not been investigated previously. Given that the existing research on this topic has taken place almost exclusively in North America, we did not have any strong a priori theoretical hypotheses on the themes that would be present in the UK master narrative for overcoming trauma and adversity. For this reason, we adopted a qualitative methodology to enable us to explore participants' beliefs about the experience of trauma and recovery process following selected national tragedies with an inductive data-driven thematic coding process, designed to avoid imposing the researchers' conceptual preoccupations on the data set.

\section{The Present Study:}

Studying the master narrative at the level of the psychology of the individual person presents some methodological challenges. In the empirical studies previously reviewed, the researchers typically used a version of the life story interview methodology developed by McAdams (2006), where participants narrate their life story as if they were writing a book with significant life experiences divided into distinct chapters in their lives. The life story interview methodology is grounded in the assumption that the salient memories that people recall about their lives link to the master narratives that shape their personal identity (Bruner, 1990). Yet, how exactly individuals' stories link to or echo the master narrative of their culture is a far more complicated methodological question. According to McLean and Syed (2016), an individual's personal life story is the product of a negotiation process between the person's own subjective reality and their interpretation of the master narrative. The first process involves an implicit embodiment of the master narratives within a culture (Hammack \& Toolis, 2015), and the second process involves the individual evaluating how connected they feel to their culture in terms of the position they embody in relation to the master narratives of their culture (Bamberg 
\& Andrews, 2004). For some, this may be a highly explicit evaluation, particularly when people feel that their experiences do not align with the master narrative, and for some the internalization of the master narrative may be implicit, necessitating different kinds of 'reads' of the narratives (McLean et al., 2018; McLean, Shucard, \& Syed, 2017).

The study of narratives can therefore be viewed as both a methodology to define cultural master narratives and as a methodology to study how cultural meanings are made and maintained within peoples' lives (McLean et al., 2017). However, this latter methodology for studying narratives benefits from knowledge of the underlying cultural expectations embedded in the master narrative to understand whether the individual is deviating from the master narrative and engaged in a process of developing an alternative narrative. McLean et al. (2017) used a narrative prompt to access narratives from people who felt their experiences did not conform to social, cultural or familial expectations. Researchers prompted participants to consider whether they had ever felt that their own experiences or interpretations of their life experiences were mismatched from what others felt was “appropriate, normal or expected” (McLean et al., 2017, p. 635). Thus, to capture the essence of a master narrative, researchers need to focus on elucidating the social context that frames the internalisation of the master narrative.

However, the methodology used by McLean et al. (2017) may be more effective when the narrative content is more obvious (e.g., life course events; McLean et al., 2019), rather than the presumed implicit nature of how to emotionally structure narratives of challenge, particularly when there is not an existing evidence base determining the content and structural form of the master narrative from which some individuals' stories may deviate. Given that there is currently to our knowledge no psychological research into the master narrative for meaning-making in the UK, we developed a novel methodology to access the master narrative. We asked individuals to narrate experiences of national tragedies from the perspective of someone directly involved in those tragedies. We used this third-person narration method, to separate the participants' own read of the cultural narrative using their personal accounts of adversity, from the shared cultural 
narrative internalisd through the participant's prior knowledge about the content and form of the master narrative that they had internalised as part of their embodiment in the socio-cultural context of the UK. We reasoned that the process of narrating a shared cultural tragedy would be most likely to tap the underlying themes in the master narrative for meaning-making in the UK, because our selected participants had not directly experienced these tragedies (and had low levels of lifetime adversity) and would therefore draw upon stories discussed more broadly in national media and by others.

Thus, in this study, we attempted to elucidate the social context of the master narrative for overcoming trauma and adversity in the UK by developing a novel method that developed and extended ideas from previous studies by Adler and Poulin (2009) and McLean et al. (2017). Specifically, we recruited participants with low exposure to trauma and adversity in their own lives and asked them to select and write about a national tragedy from the perspective of a survivor. As our participants had limited experience in managing traumatic experiences in their own lives, we reasoned that their views on the recovery process of survivors directly affected by their chosen national tragedy would be based on portrayals of recovery from popular culture, including media portrayals of the event and similar tragedies, vicarious experiences observed from the life experience of others and social and cultural norms. By asking participants to construct a vicarious mental representation of a specific national tragedy, the master narrative underpinning stories of adversity and trauma was captured in two ways; firstly, through the context of the aversive cultural tragedy, and secondly through the context of another person's understanding of overcoming this tragedy. Both these mechanisms access the emergent master narrative for recovering from trauma and adversity in the UK, internalised through the shared socio-cultural conception of how overcoming adversity should be understood in the context of these tragedies. 


\section{Method}

Participants: Data from 65 participants were analysed. We recruited individuals through opportunity sampling to participate in an online survey that examined people's perceptions of major national events that had occurred in the UK between 2005 and 2017. Participants included university students enrolled on a psychology degree at a British university who participated in exchange for course credit and members of the general public recruited through social media (i.e., Facebook and Twitter) who participated in exchange for entry into a prize draw to win 1 of $5 £ 20$ e-gift vouchers. Given that the study was exploring the master narrative for overcoming adversity in the UK, we established strict inclusion criteria a priori to data collection (see Figure 1 for a consort flow diagram outlining participant exclusions and basic demographic information).

As can be seen from Figure 1, our sample was predominantly young, female and White British. The implications of which are considered in the discussion. However, our sample was not too dissimilar in ethnicity from the national population reported in the 2011 UK census (Office for National Statistics, 2018), of which $80.5 \%$ of the population identified as White British, $4.4 \%$ as White other, 2.5\% Indian, 2\% Pakistani, 1.1\% Black Caribbean and 0.7\% Chinese. The exclusion criteria were designed to ensure that participants were born and socialised within the UK and had little experience of adversity in their own lives. We used recommendations from Seery, Holman, and Silver (2010) and classified participants as low in lifetime adversity if they had endorsed 4 or fewer events on their cumulative lifetime adversity questionnaire. It should be noted that this count measure assumes that experiencing multiple events is worse psychologically and those with the same count had similar experiences. The limitation of this count measure is that it fails to take into account the subjective evaluative impact an event has (or does not have) on an individual (Berntsen \& Rubin, 2006).

Researcher Description: The first author's research expertise is on understanding how people make meaning and change in the aftermath of highly challenging life experiences. Similarly, the fourth author's research expertise is on how people recall and interpret experiences in narrative 
form and understanding how the narrative process is affected by interactions with others and the individual's cultural context. The research expertise of these authors therefore ensured that the methodology was appropriate and suitable for our research questions. Importantly, the authors also differ in their nationalities with the first author from the UK and the second author from the US. This ensured that our methodology was situated within the existing methodologies used to study master narratives, yet was culturally appropriate for use in a UK sample. The third author's research expertise is within the area of health psychology focusing on message framing effects on health decisions and behaviours. The third author is an experienced qualitative researcher, and as well as the first author, has experience in teaching and supervising students using our chosen methodology. The second author was - at the time of data collection and analysis - a postgraduate student supervised by the first author. The second author had some knowledge of the existing research, but not sufficient engagement with and investment in the topic to bias her primary responsibility for data coding and initial theme extraction. The third author's assistance with data analysis also ensured that our themes were well grounded and supported in the data.

Materials and Procedure: The study received ethical approval by the first author's university and participants provided informed consent and were debriefed on the aims of the study after completion. The method was designed by the first, second and fourth authors. Data were collected via the online survey software Qualtrics. All the participants were provided with an anonymous link, which directed them to a screen that displayed 10 national tragedies that had occurred in the UK between the years of 2005 and 2017. Participants selected all of the national events they could recall happening or following coverage about in the media. The tragedies displayed were: 7/7 London bombings (2005), Northumbria police manhunt for armed shooter Raoul Moat (2010), the winter storms on the Somerset levels (2013-2014), Glasgow helicopter crash (2013), the public murder of British solider Lee Rigby in a terrorist attack (2013), Glasgow bin lorry crash (2014), Shoreham air show crash (2015), flash floods in Cumbria, Southern 
Scotland and parts of Ireland (2015-2016), Manchester bombings (2017) and Grenfell Tower fire (2017). The events were chosen by the second author with the intention of including a diverse range of adverse events from terrorist attacks to natural disasters. Despite the diverse nature of the presented adverse events, most participants wrote about terrorist attacks - 44 people wrote about the Manchester bombings and 3 people about the 7/7 London bombings. The other 18 participants wrote about fatal accidents that received media attention - 15 wrote about the Grenfell Tower fire, 2 people wrote about the Glasgow bin lorry crash and 1 person about the Shoreham air show crash. Thus, most of our participants wrote about the two most recent events from our given list.

On the next screen, participants were shown their selections and asked to select the one national event that they recalled the best and could answer open-ended questions about. The first 4 open-ended questions were designed to encourage participants to vividly recall the chosen event. These questions were not analysed; these were included to facilitate detailed recall and enable participants to take the perspective of a victim of this event in the following questions, and as such these 4 questions are not provided here. Participants responded to the following 8 open-ended questions, which were designed to have participants adopt the perspective of someone directly involved in their selected tragedy. We analysed responses to all 8 questions to develop our thematic map:

1. Please describe what a person directly affected by this event would find particularly challenging to deal with.

2. Do you think the challenges faced by a person directly affected by this event would change over time? If so, how do you think the challenges faced by this person would change over time?

3. How do you think a person directly affected by this event would try to cope with these challenges? 
4. Do you think other people would think that the person was handling the situation well if he/she used the coping methods you described above?

5. Do you think it is possible for a person directly affected by this event to recover from their experience?

6. If so, what does recovery look like for someone in this situation?

7. How do you think the process of recovery unfolds over time for a person directly involved or affected by an event such as this one?

8. Do you think an event like this would have a lasting impact on the person? If so, how do you think the event would change the person?

Afterwards, participants completed a series of questions assessing the exclusion criteria (see Figure 1) followed by demographic information and a lifetime adversity questionnaire (Seery et al., 2010) ${ }^{1}$, which presented 37 adverse events and asked participants to select all the events experienced throughout their whole lifetime from childhood to adulthood. Adding up the number of events endorsed by each participant creates a cumulative lifetime adversity score.

Data Analysis: The data were analysed with an inductive thematic analysis. The purpose of thematic analysis is to extract core themes that are repeated across the whole data set, to develop a framework with which to organise and interpret the data in light of the key research questions. An inductive thematic analysis involves a data-driven coding process where extracts of data relevant to the research questions are identified in a manner that stays true to the raw data and avoids imposing the researchers' conceptual preoccupations on the data set (Braun \& Clarke, 2006). We followed the 6 analytic steps outlined by (Braun \& Clarke, 2006, p.87) to adhere to their guidelines of robust analytic practice when undertaking thematic analysis:

\footnotetext{
${ }^{1}$ The authors are grateful to Mark Seery for providing them with a copy of the cumulative lifetime adversity checklist.
} 
1. Data familiarisation: repetitive reading and re-reading of the data set to familiarise ourselves with the content and noting down initial ideas that are relevant to our research questions.

2. Data Coding: systematically coding ideas across the whole data set that captures an aspect of relevance to our research questions.

3. Initial Search for themes: collating the codes to reduce repetitions and organising conceptually similar codes under potential themes.

4. Reviewing themes: checking that the themes work in relation to the coded extracts and in relation to the entire data set.

5. Defining and naming themes: revisiting the thematic map and the raw data set to refine themes and identify core conceptual ideas encapsulated by each theme.

6. Producing the report: selecting appropriate and varied quotations from the data set to demonstrate how the conceptual ideas contained in the themes manifest in the data set.

The second author initially undertook steps 1 to 3 of the analysis. For the data coding process, she identified data extracts that were relevant to the following research questions: (1) what are the challenges faced by survivors of collective tragedy? And, (2) how do participants believe survivors of collective tragedy overcome these challenges? The first and second authors decided to code the data with respect to both questions, because recovery is not a static process, and by examining both questions we were able to explore how participants perceived such processes to unfold over time from initial challenges to recovery. The data was coded by the second author at the semantic level adopting an essentialist standpoint (Braun \& Clarke, 2006) that assumes that people's language and descriptions provides direct insight into their understanding of the recovery process following experiences of trauma and adversity. The first and third author undertook steps 4 and 5 with the second author to help refine the content of the themes and resulting thematic map, and to try to ensure that the themes were not entirely 
subject to the second author's position and interaction with the data set. As with all qualitative research, researchers must carefully attend to the process of knowledge construction and their own influence on this process (Levitt et al., 2018). For this reason, we discussed and refined the thematic map as a team to use the method of triangulation to ensure our themes were grounded and well supported within the data set. Once the team had come to agreement on the content of the themes and structure of the thematic map (step 5), including the relationships between the different themes, the first author undertook stage 6 with support from the third and fourth authors.

\section{Results}

The aim of the present study was to identify the narrative themes individuals in the UK used to narrate stories of recovery from traumatic life experiences. As will be discussed later on, we identified four themes from our thematic analysis, two of which captured outcomes of recovery. Our analysis also identified a dominant pathway in which many of our participants outlined how they believed survivors could achieve recovery. For this reason, we extended our analysis to explore which of the two themes was more prevalent in the data set. Based on McLean and Syed (2016), we reasoned that the prevalence of the two recovery themes would give an indication of which theme would be more likely to characterise the master narrative for meaning-making following trauma in the UK. This secondary research question resulted from the results of our thematic analysis. We have therefore organised our results into two separate sections to clearly distinguish results pertaining to each of these research questions.

\section{$\underline{\text { Thematic Analysis }}$}

We identified four themes from our thematic analysis: (1) adverse reactions to trauma with the following three sub-themes: (i) psychological pain and physical suffering, (ii) altered perceptions of self and others, (iii) maladaptive coping strategies (2) active coping strategies, (3) 
recuperation and (4) redemption. We will describe each of the themes in turn and provide supporting evidence from participants' data. Following thematic analysis reporting procedures of Clarke and Smith (2015), we have corrected any spelling and grammatical errors in our presented data extracts to aid readability and participants are referred to by an ID number in the presented extracts to protect their identity.

(1) Adverse Reactions to Trauma: This overarching theme captured the psychological struggles, physical health issues, and the social and economic challenges participants believed survivors directly affected by these national tragedies would experience. The challenges were quite varied, therefore we further organised the ideas into the following three sub-themes. All the codes encapsulated in a theme or sub-theme are presented in Table 1.

(A) Psychological Pain and Physical Suffering: participants discussed the difficulties individuals directly affected by these national tragedies would struggle to deal with. In some cases, participants identified issues survivors of these tragedies would struggle with in the immediate aftermath. These issues included symptoms of PTSD (including anxiety, intrusive memories and flashbacks), difficulties sleeping, feelings of anger and resentment at those responsible for the tragedy and survivor's guilt.

"They may struggle to go to any events similar to this in the future, be anxious and scared around large crowds and may still get frequent intrusive and scary memories about the event." (S\#7).

"Guilt - could I have done more to save people in the block... Pain, potentially nightmares, flashbacks. People may feel outraged and angry at the people involved in the building design for letting something like this easily happen." ( $(\$ 40)$

"I think they would particularly struggle with thinking that how fortunate it was that those seriously injured could have easily been them and would feel and extreme sense of guilt as it's so unfortunate for those who were seriously injured." (S\#14)

However, participants also acknowledged that there were some challenges that victims may continue to struggle with. This is not to say that participants felt recovery from these issues was 
impossible, rather that it would take some time and require support. The most prominent and persistent struggles identified by participants were depression, grief from losing a loved one or valued social possessions (e.g., housing), debilitating physical injuries, and seeing offers of social support diminish over time.

"Grief if [they] lost a loved one. [It] doesn't get easier, it just comes in waves, so sometimes they could be feeling distraught and angry, but other times they might be able to deal with it." (D\#3)

"If they were living in the tower block for example, they may have to deal with burn injuries if they got in the direct path of the fire. They may also have to deal with loss of friends, family, and neighbours, as well as the loss of their homes and possessions. These I think would be the most challenging obstacles to overcome, based on my best guesses and not having personally lived through such an event myself." (D\#19)

"I have realised the psychological effects were more complex, widespread and life changing for so many. Even the physical injuries still persist so long after; it must be so borrific." (S\#2)

"I think the wave of public sympatby would be supportive initially, but after a few years, as people forget and other news items and sad happenings take their place, this would dissipate, and people may begin to feel isolated." (D\#16)

(B) Altered Perceptions of Self and Others: In this theme, participants discussed how a conditioned fear response from the tragedy may continue to shape the survivor's perspective of themselves, others, and their social environment. Participants wrote about how such tragic experiences can leave survivors with an ingrained fear that it could happen again at any time, which in turn can lead victims to avoid going to similar events or crowded places in the future. Participants also discussed how this conditioned fear would resurface when they heard about similar events happening elsewhere from the media or when they felt unsafe in public places.

"I think it could always be nerve wrecking when getting into a crowd of people as you can never truly know what a person has on them." (D\#1)

"Any time the news reported further terrorist attacks, I think this would further reinforce the person's feelings of fear." (S\#8)

"They would find it difficult to deal with the news constantly reminding them of the event ... They would find it difficult to go outside (maybe) as they'd feel scared knowing that such horrible people existed who are capable of such horrible acts" (D\#8) 
Due to this heightened fear, participants discussed how the event could result in lasting negative personality changes, including encouraging survivors to be more cautious, introverted, protective of their loved ones, especially children, and be more distrustful of other people.

"I also think that if they ever saw someone acting vaguely suspiciously they would become very anxious and would struggle not to see the worst in people. In addition to this, if for example they were a parent, I believe they would become very controlling over their children in order to avoid such things happening again and they would be very over protective." (S\#8)

"Of course, this would have an impact on the people that were there. I think this may make an outgoing person possibly very quiet and make them not want to go out like they used to." (S\#17)

"The sense of order and safety would be broken. How would you feel safe on the transport system again? Things you had always taken for granted would be uprooted. Could somebody you know be a terrorist? How could you identify which people were involved? ... A whole new murky view of humanity starts to take shape" (D\#16)

(C) Maladaptive Coping Strategies: Given the severity of the tragedies and the resulting challenges, participants discussed how some survivors may try to manage their negative feelings by relying on unhealthy and self-destructive behaviours. These strategies included survivors bottling up their feelings and avoiding talking about the event, isolating themselves from others, blaming themselves for being in the wrong place at the wrong time, engaging in self-harm behaviours and substance abuse.

"For some victims, if they blame themselves they could turn to self-harm as a way of coping with pain." (S\#40)

"They may turn to drink or drugs to blot it out [...] They may become reclusive to avoid such a situation bappening again." (D\#10)

"Someone who surrounded themselves with family and friends and took some time off work to recover would probably be judged as handling the situation well. Whereas, someone isolating themselves and working longer hours or turning to alcohol/ drugs would be judged as handling the situation poorly." (S\#41)

"I think if they talked about it and got help their [symptoms] would decrease but if they kept it to themselves their fears and worries may get worse." (D\#7) 
(2) Active coping strategies: This theme encapsulated a broad range of coping strategies that participants viewed as adaptive ways for survivors to manage their feelings and try to recover from the trauma and resulting challenges. Although the coping strategies were broad and varied in nature (see Table 1), all the strategies detailed a pathway to recovery as involving self-directive activities, where the survivor decided to engage in proactive thoughts patterns and behaviours or actively sought support from other people. Many of the ideas captured the different ways survivors could seek social support from others. For example, participants discussed how survivors could talk to their loved ones to help manage the emotional impact of the event, attend victim support groups, turn to their faith for emotional support, and seek out professional support from medical and mental health specialists, including medication and counselling services.

"They would most likely call on their network of friends and family for emotional support. They may seek therapy to cope with the event." (D\#11)

"Some people may go to support groups where they can meet other people affected by the same or similar events, which might help them to make friends and get other people's views/perspectives on how they cope with their own challenges. Another method could be turning to religion - for example, praying." (S\#22).

"Counselling, therapy, medication, physiotherapy, care assistant, home adaptations" (S\#10)

Other coping strategies identified by participants focused on how survivors could actively try over time to change the negative and maladaptive perceptions that had developed in response to the event. These strategies were again varied, and some involved victims changing how they thought about tragedy and their future. For example, these strategies involved victims reassuring themselves that the event was a rare occurrence and unlikely to happen again and learning not to blame oneself for being in the wrong place at the wrong time. Participants acknowledged the need for time in these processes, explaining how it was a gradual process where victims learned to accept it had happened, tried to adopt a positive outlook moving forward and took steps to challenge the ingrained fear response by returning to normal activities. 
"Their fears of the event may decrease overtime as they realise that attacks are a rare occurrence in their everyday life, however this may take a long time for the thoughts of the Manchester bombings to leave their mind." (D\#22)

"Perhaps in time, someone may learn not to blame themselves, they may find it hard to think what life could be like now, had they not attended the concert." (S\#34)

"They would try to cope by thinking positively in the sense, that a lot worse could've happened, but thankfully it didn't. Also, by looking at a brighter future and just thinking of a more positive outlook." (S\#11).

They aren't fearful of going out to places and are accepting of what has happened and feel strong that they survived." (S\#14).

(3) Recuperation: Most participants believed that recovery from tragedy was possible, but they acknowledged that it was a gradual process that unfolded over time. In our sample, recovery was viewed as a gradual lessening in the impact of symptoms over time and an ability to return to normal everyday activities while continuing to manage the impact of any lasting emotional and physical scars. Thus, recovery was not seen as a simple reversion to a former pretrauma life. Participants acknowledged that this was impossible, as victims would have lost loved ones and suffered a trauma, they were unable to forget. Instead, participants thought that recovery was when a person did not let the memory of the event direct future decisions and they felt safe to return to everyday activities or when they could remember a loved one fondly without feeling overwhelmed by grief.

"I think time really helps come to terms with losing someone close to you, even though they will probably never get over it, the grief will get easier to deal with. As time goes on, people would be more focused on rebuilding their lives and trying to return to normal." (S\#37).

"I think recovery looks like not forgetting about what happened but moving on from it and not letting thoughts about what happened control your life." (S\#44)

'I don't think a full recovery in a sense will ever be made, because they will always have the memory of the event and potential grief of losing someone. They could definitely recover from physical injuries that occurred from the bomb detonation, but although they will find more effective ways to psychologically cope with the event, they will never be able to forget it happened [...] I think physical recovery would be a reversion to the individual's optimal state with everything working as it should be. Psychological recovery will depend on the individual and may be something like talking about the event to someone, being able 
to trust in others and express feelings or going from feeling unsafe in the house to attending another concert. It's all about being able to emotionally deal with what happened and almost move past it, preventing it impacting the day to day activities an individual would wish to engage in." (S\#20)

(4) Redemption: Some participants discussed how recovery may over time become more than a return to normal life and an effort to minimise the impact of emotional and physical scars. In contrast to ideas presented in the altered perceptions of self and others theme, participants acknowledged that survivors could sometimes identify insights from surviving tragedy, which could positively shape their lives moving forward. These involved a heightened awareness of the fragility of life, which resulted in appreciating life and not taking it for granted. Participants also identified how tragedy might positively shape the victim's personality, as well as outlook on life. It was thought that through the process of overcoming tragedy, victims might develop a greater sense of personal strength, equipping them with coping skills to effectively handle challenges they may face in the future. Additionally, participants discussed how given the victims' own experiences, they might become more sensitive and empathetic to others who are struggling in times of hardship.

"I think it would change a person's perception on what is important in life." (D\#14)

"People involved in these sorts of events will always change their perspective on life. I believe that those who were affected will be very grateful for life and have the view that it needs to be lived to the maximum as you never know when certain events may occur, and it may be your last day. I think the events will also change people's perception of how to handle such situations; by overcoming certain feelings of sadness and grief and wanting to make a difference by showing others that they will not be affected and are instead a stronger person because of it." (S\#26).

"The person would change as they would be grateful for those around them and their lives, acknowledging that life too short to remain angry at the world." (S\#28)

"Yes, it might make them more wary and anxious of big events like these and more empathetic to those who have been in a similar situation as they know what it's like." (S\#10). 


\section{The Prevalence and Pathway to Recovery after Trauma:}

We identified two themes in our thematic analysis that detailed how participants believed survivors could achieve recovery from trauma: recuperation and redemption. We were therefore interested to further explore whether redemption was the dominant response in our UK sample, as it is proposed to be in the USA (McAdams, 2013). To do this, we examined the prevalence of the two themes in our data set. We gave a research assistant who was blind to our study aims all 65 narratives and our definitions for the recuperation and redemption themes. We asked her to code for the presence or absence of recuperation and redemption in each narrative. We wanted to quantify the prevalence of these two themes in our sample to determine whether either one of these themes was more prevalent than the other. We reasoned that if one theme was more prevalent than the other, it would suggest that this theme is a more central idea in the UK master narrative for overcoming adversity in the UK.

In our sample, recuperation was found to be the most prevalent outcome with 63 out of 65 participants $(97 \%)$ discussing recovery from tragedy as involving a reduction of symptoms and an eventual return to everyday activities. In contrast, we found only 21 out of 65 participants $(32 \%)$ reported redemption when discussing how survivors recover from trauma. It is interesting to note that these two recovery themes were not mutually exclusive from one another. However, participants' narratives did not specify the relationship between these themes, rather redemption was discussed by some participants, but they did not explain whether recuperation was necessary prerequisite for redemption. Participants' narratives however, did outline a clear pathway for how recuperation was achieved. As indicated on the thematic map (Figure 2), participants indicated in their written responses that recuperation involved three themes: moving away from the adverse reactions to trauma to recovery by adopting active coping strategies. This pattern in the narratives was noted in discussions between the first and third authors. The first author reread all 65 narratives and counted the frequency with which this pathway was endorsed. We found the pathway to recuperation was endorsed by 51 of the 65 participants $(78 \%$ of our 
sample). Thus, the three themes were connected in most of the participants' data and outlined the process individuals are expected to undergo to recover from traumatic life experiences. Our results therefore suggest that this pathway to recuperation may underpin the master narrative for how people are expected to manage and overcome trauma in the UK.

\section{Discussion:}

The aims of the present study were to identify the key narrative themes that characterise the UK master narrative for overcoming traumatic life experiences. We were interested to see if redemption was a dominant theme to emerge from the data, because this theme has been found to be prevalent in North American narratives of adversity (McAdams, 2013). We undertook a thematic analysis on participants' responses to open-ended questions that asked them to outline how a survivor of a specific national tragedy would react and how they could try to come to terms with their experiences. As outlined in the method section, the participants were carefully selected to ensure that they had limited personal experience in managing adversity in their own lives and were born and raised in the UK.

Participants identified two ways in which survivors could overcome trauma: recuperation and redemption. Recuperation was most commonly reported, and consistent with medical definitions of recovery (Schrank \& Slade, 2007) it was described as a gradual lessening of the impact of symptoms over time and the ability to manage the impact of lasting emotional and physical scars. Similar to previous research (McLean \& Breen, 2009; McLean \& Lilgendahl, 2008; McAdams et al., 2001), redemption captured survivors' ability to take some good from otherwise tragic and highly challenging situations (e.g., appreciation of life and sense of personal strength). However, many in the sample did not report redemption, instead participants were more likely to discuss the pathway survivors could take to attain recuperation. This pathway involved survivors' moving from the emotional pain of the tragedy to recuperation through the adoption of active coping strategies. The coping strategies were varied, but importantly they all involved survivors 
deliberately choosing to engage in proactive thoughts and behaviours to accept and adapt to their post-trauma reality. Thus, our results suggest that this recuperation pathway might be reflective of the content and structural form of the master narrative for overcoming trauma in the UK.

Research by sociologist Furedi (2007a) supports our findings that recuperation might be integral to the UK master narrative for managing adversity. Furedi's (2007a) research compared responses from UK newspapers to two national flood disasters in the UK in 1952-53 and 2000, concluding that cultural conceptualisations for managing adversity have shifted from a narrative of community resilience to a narrative of vulnerability. He argued the 'Blitz spirit' characterized media responses to the flood disasters in Britain in the 1950s, whereby the legacy of WWII promoted resilience, resourcefulness and adaptability and communities rallied together to return to normality quickly. In contrast, he argues the narrative of vulnerability is characterised by an increasing focus of the negative impact of adversity on the identity of the individual. Furedi (2007a) demonstrated that media representations of the flood disasters in Britain in 2000 were understood in the context of individual stories of trauma, and as a consequence the management of adversity was focused on providing support for individuals to cope with the long-term (and possibly irreversible) repercussions of post-disaster life.

We found evidence in support of the narrative of vulnerability, as our participants viewed recuperation in terms of an individual's own ability to seek support to manage their emotional trauma and physical pain. There was similarly an acknowledgment of the long-term impact of trauma on survivors' identity, as participants recognised that survivors may not be the same person as before, even after they had returned to their normal activities. However, while our results align with Furedi's (2007a) analysis of the shift in the cultural representations of adversity within the UK over time, Furedi (2007b) did not necessarily view the vulnerability narrative as unique to Britain. He writes "Western culture frequently transmits the view that we live in a uniquely dangerous era where humanity faces hazards and potential disaster" (p.485), which he 
argues is the underlying ideology of the narrative of vulnerability; that we, as humans, are inherently vulnerable. He claims that this narrative shift is underpinned by changes that occurred in the 1980s to our understanding and approach to mental health.

Thus, it is certainly possible that recuperation might also be observed in other Western cultures, such as the US. This possibility is further supported by contemporary theories from clinical psychology. For example, Janoff-Bulman's (2010) theory of shattered assumptions posits that trauma can overwhelm an individual's ability to cope, leaving them vulnerable and liable to question previously-held implicit assumptions about the safety and benevolence of the world. It is these shattered assumptions that if left untreated may lead to clinical diagnoses, such as PTSD. Janoff-Bulman (2010) argues that the treatment of trauma requires helping the individual to rebuild or modify their shattered assumptions, enabling them to return to their normal everyday lives. This notion of supporting an individual to process and overcome the negative impact of the trauma is therefore not dissimilar from the narrative of vulnerability proposed by Furedi's (2007a).

Thus, to fully address the question of whether recuperation is a UK master narrative or not, we need to undertake a dedicated programme of research with an interdisciplinary team of scholars. The first step in this programme would be to determine if our findings replicate in the personal narratives of individuals who had experienced tragedy first-hand. If recuperation is a relevant cultural master narrative in the UK, then we should see this narrative in people's own stories, and moreover it should predict greater adjustment to adversity. Simultaneously, scholars in sociology, English literature and media studies would need to analyse the degree to which this narrative was represented in popular culture (e.g., media coverage, fiction and films). Finally, we would need to conduct a cross-cultural analysis of the prevalence and function of recuperation (compared to redemption) in other Western cultures, such as the UK and US focusing on a broad range of sources (e.g., personal narratives, fiction, films and media coverage of relevant national tragedies). 
Interestingly, although we found evidence for redemption in our analysis, the expression of this idea differed significantly to the manner in which it has been conceptualised in existing research. For example, McAdams (2006, p.81) has defined redemption as "the deliverance from suffering to an enhanced status or position in life". The narrative coding scheme used to capture redemption in individuals' life stories is therefore focused on whether an emotionally negative life scene is transformed into a positive life scene. In our data, a small number of participants commented on how survivors of tragedy might report some positive changes to their outlook on life and personality. However, in contrast to the existing definition of redemption (McAdams, 2006), participants did not view these positive outcomes as redeeming narrative features that salvaged the person from their suffering. Instead, participants narrated the lasting impact of tragedy to be more emotionally mixed, with positive and negative outcomes co-existing within the survivor. For example, one participant when asked whether there would be a lasting impact of the event said:

"The impact would be long lasting, it will make the person feel more thankful for life and appreciate the small things and not take life for granted. They may also be put off from going to places and doing things as a result of being scared." (S\#7)

Another participant described the lasting impact on survivors of tragedy as:

'I think there will definitely be a lasting impact, but it doesn't necessarily have to be a negative one. By this I mean an individual may adopt the attitude of taking every day as it comes and living their life more proactively than they once did, expressing gratitude for the small things they may have previously overlooked. Potentially, they will have a more nervous outlook on attending certain events and engaging in experiences as there will be a sense if fear that something will happen again.” (S\#20)

Thus, while redemption was present, it did not appear to function to salvage the negativity of the event, as conceptualised by McAdams (2013). It is unclear why this is the case or whether it is an artefact of the severity of the national tragedies participants decided to write about. However, it is interesting to note that Adler and Poulin (2009) found evidence of redemption, as traditionally conceptualised by McAdams (2013) when asking participants in the US to write narratives about 
the $9 / 11$ terrorist attacks. Similar to our study, their participants were also largely not directly involved in the terrorist attacks.

Our study is not without its limitations and these issues need to be carefully considered in future research. First, our participants were presented with a diverse range of adverse national events, including natural disasters and accidents. However, the majority of participants chose to write about terrorist attacks (i.e., London bombings in 2005 or Manchester bombings in 2017) or the Grenfell Tower fire in 2017. For all of these events people perceive there to be a culpable party, with local government authorities widely publicly blamed for the lives lost in the Grenfell Tower fire due to failures to replace building cladding that did not meet fire safety standards. It is unclear from our data set whether recuperation would be a central theme in narratives in response to a broader range of adverse and traumatic events, where a culpable party is harder to identify.

Second, our sample was mostly young White British females enrolled in university. It is important to note that master narratives are upheld by the privileged, who are more likely to align with them, and perhaps also do reflect them in their reports (McLean \& Syed, 2016; McLean et al., 2017). However, those who do not align can also help researchers to see what the master narratives are - the deviation reveals the expectation (e.g., McLean et al., under review). Thus, to draw stronger conclusions on the content and structural form of the UK master narrative, we need a more balanced sample, where a range of different genders and ethnicities are represented. Our data only suggests one plausible narrative, and we know from existing research that people differ greatly on the extent to which they deviate from a master narrative (McLean et al., 2017).

Third, we used strict a priori exclusion criteria to select participants who would be most likely to report on the UK master narrative for overcoming trauma. We selected participants born and raised in the UK who had not spent any prolonged time outside the UK and had little experience in managing adversity in their own lives. However, with the rise of popular media, it 
is impossible to truly know which cultures and sources our participants drew on to outline their expectations for survivors' recovery experiences. Additionally, given the rise of popular media, it is possible that some participants were drawing upon American ideals, especially when discussing notions of redemption. Many participants selected to write about the Manchester bombings in 2017, which took place during a concert by an American signer - Ariana Grande. This event was reported in the global media, and the public response was likely partly shaped by this fact. For example, one year after the attack, there was a Manchester Together concert where survivors and singer Ariana Grande came together as a show of strength and resilience. This event embodies the notion of the master narrative of redemption in North America (McAdams, 2006). To address this issue we need to take an interdisciplinary approach to this type of question. Specifically, scholars from humanities and cultural studies are needed to analyse the dominant social-cultural narratives underlying popular UK culture, while psychologists continue to collect personal narratives from people about their own experiences to determine whether recuperation appears organically in people's own narratives.

Finally, in our instructions we asked participants to write about how "the people directly involved and/or directly affected by this event (e.g., survivors or friends/family of loved ones) handled the event". While we tried to be careful to avoid influencing participants, our use of the term survivor fits with a well-known and publicly endorsed victim-to-advocate narrative (Delker, Salton, \& Mclean, under review). The narrative progression from victim to survivor is consistent with a redemptive story, and as such may have prompted redemptive story telling from our participants. However, given the relatively low prevalence of the redemption theme reported in our sample, it is unlikely that this language biased our findings too significantly in this study. Yet, it is also possible that redemption would be reported to a greater degree if participants had been asked to imagine how they would react to similar national tragedies. As discussed previously, future research needs to examine whether recuperation remains a dominant script in UK culture when stories are told from a first-person perspective. 
In conclusion, our study findings suggest that contrary to research from North America, redemption is not necessarily the underlying cultural script used in the UK for guiding meaningmaking processes in the aftermath of trauma. Instead, our study findings suggest that notions of recuperation may characterise individuals' from the UK narratives of trauma, where recovery is viewed as a long-term process of symptom remission to move past the negativity of the tragedy and return to normal everyday activities. However, to our knowledge, this is the first exploratory study within narrative psychology to examine the content and form of the master narrative for overcoming adversity in the UK, and as such, these findings await replication within a dedicated programme of interdisciplinary research. 


\section{References:}

Adler, J. M., Lodi-Smith, J., Philippe, F. L., \& Houle, I. (2016). The Incremental Validity of Narrative Identity in Predicting Well-Being: A Review of the Field and Recommendations for the Future. Personality and Social Psychology Review, 20(2), 142-175. https://doi.org/10.1177/1088868315585068

Adler, J. M., \& Poulin, M. J. (2009). The Political Is Personal: Narrating 9/11 and Psychological Well-Being. Journal of Personality, 77(4), 903-932. https://doi.org/10.1111/j.14676494.2009.00569.x

Adler, J. M., Turner, A. F., Brookshier, K. M., Monahan, C., Walder-Biesanz, I., Harmeling, L. H., ... Oltmanns, T. F. (2015). Variation in narrative identity is associated with trajectories of mental health over several years. Journal of Personality and Social Psychology, 108(3), 476-496. https://doi.org/10.1037/a0038601

Bamberg, M., \& Andrews, M. (2004). Considering Counter-Narratives: Narrating, resisting, making sense. Amsterdam, The Netherlands: John Benjamins Publishing.

Blackie, L. E. R., Cozzolino, P. J., \& Sedikides, C. (2016). Specific and Individuated Death Reflection Fosters Identity Integration. PLOS ONE, 11(5), e0154873. https://doi.org/10.1371/journal.pone.0154873

Berntsen, D., \& Rubin, D. C. (2006). The centrality of event scale: A measure of integrating a trauma into one's identity and its relation to post-traumatic stress disorder symptoms. Behaviour research and therapy, 44(2), 219-231.

Braun, V., \& Clarke, V. (2006). Using thematic analysis in psychology. Qualitative Research in Psychology, 3(2), 77-101. https://doi.org/10.1191/1478088706qp063oa

Breen, A. V., \& McLean, K. C. (2017). The intersection of personal and master narratives: Is redemption for everyone? In B. Schiff, A. E. McKim, \& S. Patron (Eds.), Life and narrative: The risks and responsibilities of storying experience (pp. 197-214). New York: Oxford university Press. 
Bruner, J. S. (1990). Acts of Meaning. Cambridge, Massachusetts: Harvard University Press.

Clarke, V., \& Smith, M. (2015). "Not Hiding, Not Shouting, Just Me”: Gay Men Negotiate Their Visual Identities. Journal of Homosexuality, 62(1), 4-32. https:/ / doi.org/10.1080/00918369.2014.957119

Delker, B., Salton, R., \& McLean, K. C. (2019). Giving voice to silence: Empowerment and disempowerment in the developmental shift from trauma 'victim' to 'advocate. Journal of Trauma and Dissociation. https://doi.org/10.1080/15299732.2019.1678212

Dunlop, W. L., \& Tracy, J. L. (2013). Sobering stories: Narratives of self-redemption predict behavioral change and improved health among recovering alcoholics. Journal of Personality and Social Psychology, 104(3), 576-590. https://doi.org/10.1037/a0031185

Furedi, F. (2007a). From the Narrative of the Blitz to the Rhetoric of Vulnerability. Cultural Sociology, 1(2), 235-254.

Furedi, F. (2007b). The changing meaning of disaster. Area, 39(4), 482-489.

Hammack, P. L. (2008). Narrative and the Cultural Psychology of Identity. Personality and Social Psychology Review, 12(3), 222-247. https://doi.org/10.1177/1088868308316892

Hammack, P. L., \& Toolis, E. E. (2015). Putting the Social into Personal Identity: The Master Narrative as Root Metaphor for Psychological and Developmental Science. Human Development, 58(6), 350-364. https://doi.org/10.1159/000446054

Janoff-Bulman, R. (2010). Shattered Assumptions. Simon and Schuster.

Lerner, M. J. (1980). The Belief in a Just World: A Fundamental Delusion. Boston, MA: Springer US. https://doi.org/10.1007/978-1-4899-0448-5_2

Levitt, H. M., Bamberg, M., Creswell, J. W., Frost, D. M., Josselson, R., \& Suárez-Orozco, C. (2018). Journal article reporting standards for qualitative primary, qualitative metaanalytic, and mixed methods research in psychology: The APA Publications and Communications Board task force report. American Psychologist, 73(1), 26-46. http://dx.doi.org/10.1037/amp0000151 
McAdams, D. P. (1996). Personality, Modernity, and the Storied Self: A Contemporary Framework for Studying Persons. Psychological Inquiry, 7(4), 295-321. https://doi.org/10.1207/s15327965pli0704_1

McAdams, D. P. (2006). The Redemptive Self: Generativity and the Stories Americans Live By. Research in Human Development, 3(2-3), 81-100. https://doi.org/10.1080/15427609.2006.9683363

McAdams, D. P. (2013). The Redemptive Self: Stories Americans Live By - Revised and Expanded Edition. New York: Oxford University Press, USA.

McAdams, D. P., \& Guo, J. (2015). Narrating the Generative Life. Psychological Science, 26(4), 475483. https://doi.org/10.1177/0956797614568318

McAdams, D. P., Reynolds, J., Lewis, M., Patten, A. H., \& Bowman, P. J. (2001). When bad things turn good and good things turn bad: Sequences of redemption and contamination in life narrative and their relation to psychosocial adaptation in midlife adults and in students. Personality and Social Psychology Bulletin, 27(4), 474-485.

https://doi.org/10.1177/0146167201274008

McLean, K. C., \& Syed, M. (2016). Personal, Master, and Alternative Narratives: An Integrative Framework for Understanding Identity Development in Context. Human Development, 58(6), 318-349. https://doi.org/10.1159/000445817

McLean, K. C., \& Breen, A. V. (2009). Processes and content of narrative identity development in adolescence: Gender and well-being. Developmental Psychology, 45(3), 702-710. https://doi.org/10.1037/a0015207

McLean, K. C., Fordham, C., Boggs, S., Byers, S., Haraldsson, K., Lowe, L., Syed, M. (2019). Personal Identity Development in Cultural Context: The Socialization of Master Narratives about the Gendered Life Course. International Journal of Behavioral Development. https://doi.org/10.1177/0165025419854150 
McLean, K. C., \& Lilgendahl, J. P. (2008). Why recall our highs and lows: Relations between memory functions, age, and well-being. Memory, 16(7), 751-762. https://doi.org/10.1080/09658210802215385

McLean, K. C., Lilgendahl, J. P., Fordham, C., Alpert, E., Marsden, E., Szymanowski, K., \& McAdams, D. P. (2018). Identity development in cultural context: The role of deviating from master narratives. Journal of Personality, 86(4), 631-651. https://doi.org/10.1111/jopy.12341

McLean, K. C., Shucard, H., \& Syed, M. (2017). Applying the Master Narrative Framework to Gender Identity Development in Emerging Adulthood. Emerging Adulthood, 5(2), 93-105. https://doi.org/10.1177/2167696816656254

McLean, K. C., \& Thorne, A. (2003). Late adolescents' self-defining memories about relationships. Developmental Psychology, 39(4), 635-645. https://doi.org/10.1037/00121649.39.4.635

National Office of Statistics (2018). UK 2011 Census. Population of England and Wales. https://www.ethnicity-facts-figures.service.gov.uk/uk-population-by-ethnicity/nationaland-regional-populations/population-of-england-and-wales/latest Retrieved from 21 October 2019

Park, C. L. (2010). Making sense of the meaning literature: An integrative review of meaning making and its effects on adjustment to stressful life events. Psychological Bulletin, 136(2), 257-301. https://doi.org/10.1037/a0018301

Schrank, B., \& Slade, M. (2007). Recovery in psychiatry. Psychiatric Bulletin, 31(9), 321-325. https://doi.org/10.1192/pb.bp.106.013425

Seery, M. D., Holman, E. A., \& Silver, R. C. (2010). Whatever does not kill us: cumulative lifetime adversity, vulnerability, and resilience. Journal of Personality and Social Psychology, 99(6), 1025-1041. http://dx.doi.org/10.1037/a0021344 
Singer, J. A. (2004). Narrative Identity and Meaning Making Across the Adult Lifespan: An Introduction. Journal of Personality, 72(3), 437-460. https://doi.org/10.1111/j.00223506.2004.00268.x

Slotter, E. B., \& Ward, D. E. (2015). Finding the silver lining: The relative roles of redemptive narratives and cognitive reappraisal in individuals' emotional distress after the end of a romantic relationship. Journal of Social and Personal Relationships, 32(6), 737-756. https://doi.org/10.1177/0265407514546978

Thorne, A., \& McLean, K. C. (2003). Telling traumatic events in adolescence: A study of master narrative positioning. In R. Fivush \& C. Haden (Eds.), Autobiographical memory and the construction of a narrative self: Developmental and cultural perspectives (pp. 169-185). Mahwah, NJ: Lawrence Erlbaum Associates, Inc.

Thorne, A., McLean, K. C., \& Lawrence, A. M. (2004). When Remembering Is Not Enough: Reflecting on Self-Defining Memories in Late Adolescence. Journal of Personality, 72(3), 513-542. https://doi.org/10.1111/j.0022-3506.2004.00271.x 
Figure 1: Consort flow diagram showing participant recruitment, exclusions and demographic information

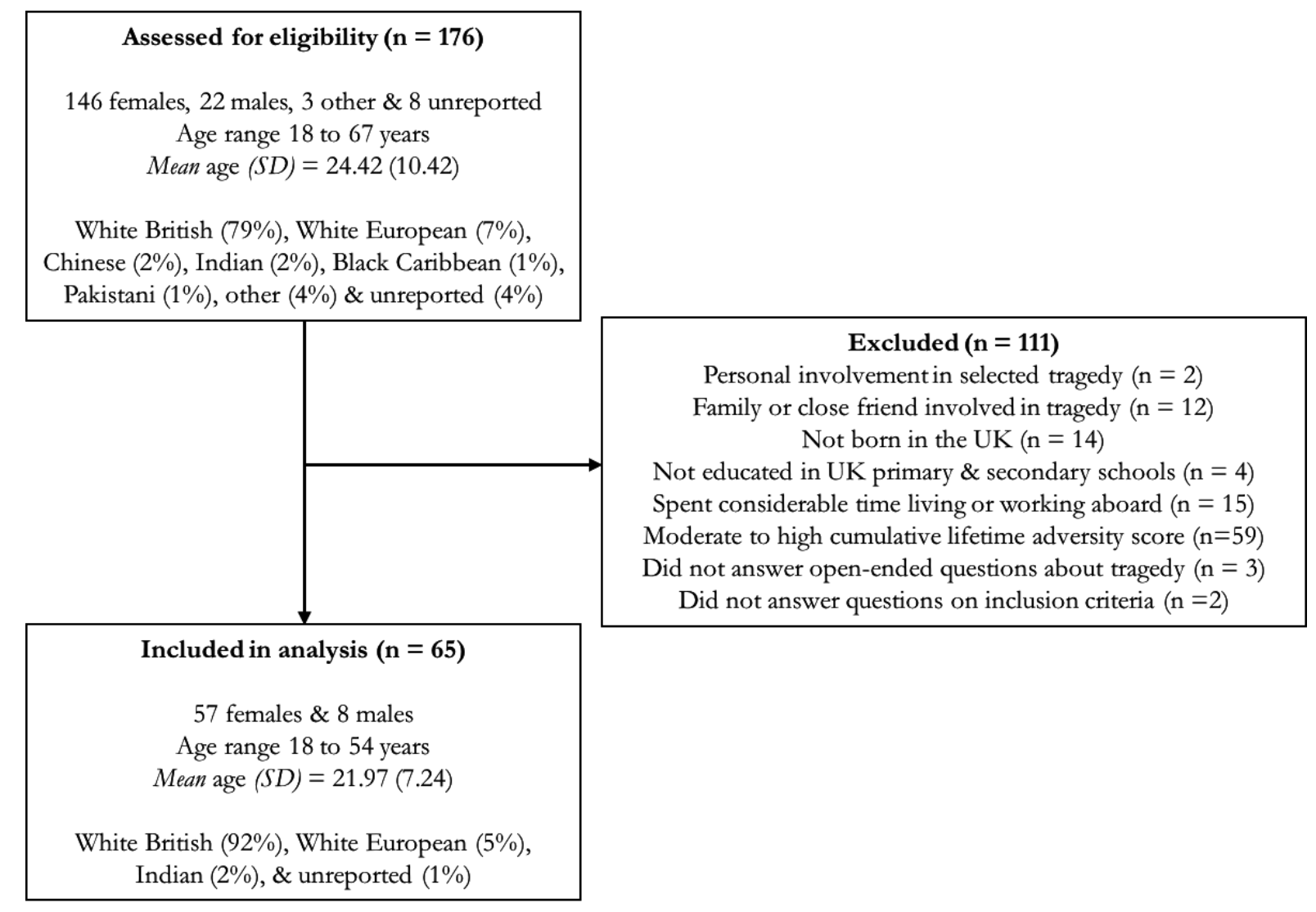


Figure 2: Thematic map showing themes and subthemes

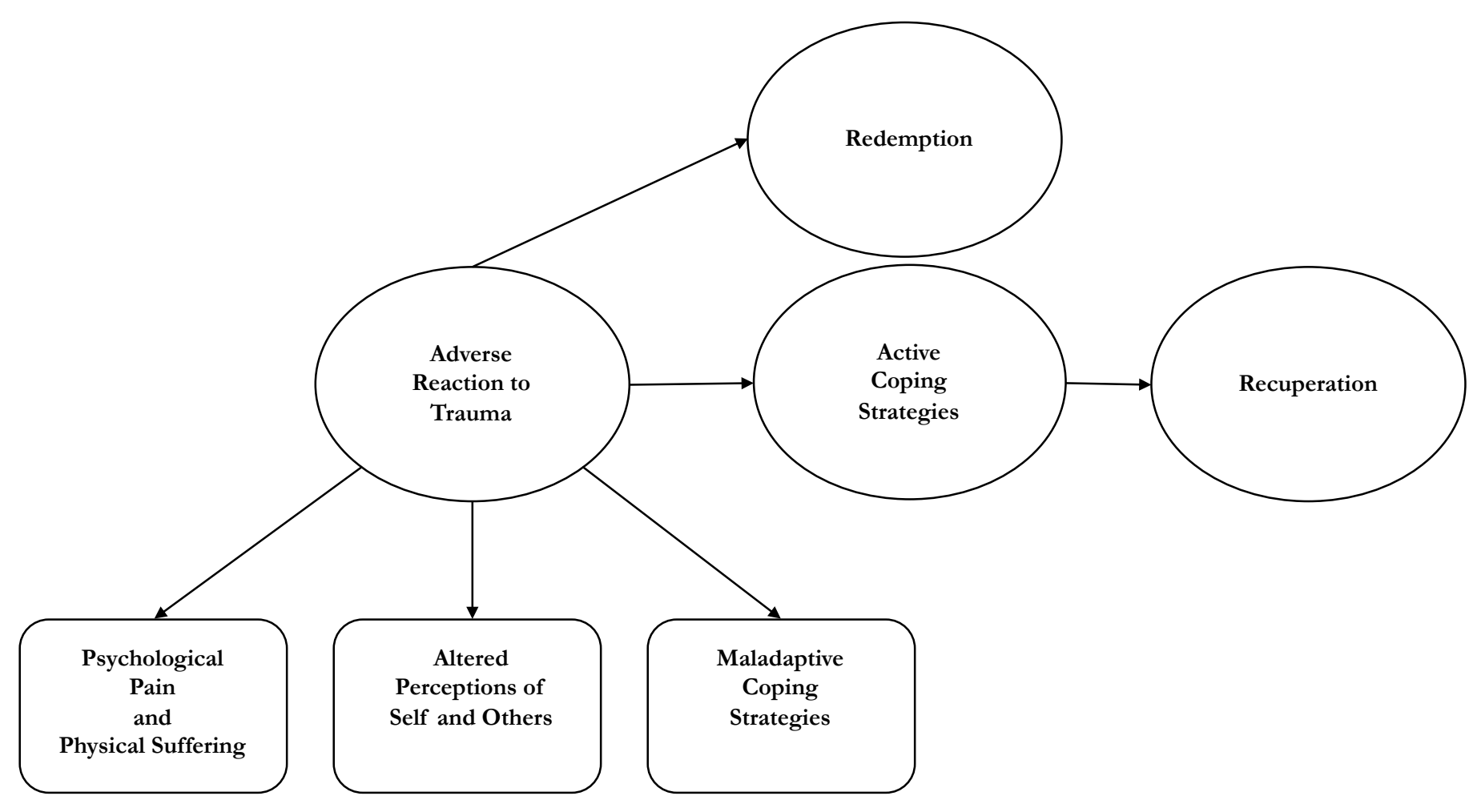


MASTER NARRATIVE FOR OVERCOMING TRAUMA IN UK

Table 1. Codes relating to each of the themes and subthemes

\begin{tabular}{|c|c|c|c|c|c|}
\hline & Idverse Reactions to traum & & Active Coping Strategies & Recuperation & Redemption \\
\hline $\begin{array}{l}\text { Psychological Pain and } \\
\text { Physical Suffering }\end{array}$ & $\begin{array}{l}\text { Altered Perceptions of } \\
\text { Self and Others }\end{array}$ & $\begin{array}{c}\text { Maladaptive Coping } \\
\text { Strategies }\end{array}$ & & & \\
\hline $\begin{array}{l}\text { Symptoms of PTSD (incl. } \\
\text { anxiety \& uncontrollable } \\
\text { flashbacks) } \\
\text { Sadness and/or depression } \\
\text { Grief over lost loved ones } \\
\text { Intrusive memories about } \\
\text { the event } \\
\text { Difficulties sleeping and } \\
\text { nightmares } \\
\text { Loss of loved ones and/or } \\
\text { possessions } \\
\text { Survivor's guilt } \\
\text { Debilitating Physical } \\
\text { injuries } \\
\text { Anger directed at those } \\
\text { responsible } \\
\text { Resentment directed at } \\
\text { those responsible } \\
\text { Offers of social support } \\
\text { can diminishes over time }\end{array}$ & $\begin{array}{l}\text { Lasting phobias about } \\
\text { crowded places } \\
\text { Desire to avoid } \\
\text { circumstances similar to } \\
\text { the event } \\
\text { Feeling broken or in a } \\
\text { permanent state of } \\
\text { victimhood } \\
\text { Feeling unsafe in public } \\
\text { spaces } \\
\text { Distrust of people } \\
\text { Fear that it could happen } \\
\text { again/ feeling paranoid } \\
\text { about possibility } \\
\text { Becoming more } \\
\text { introverted and cautious } \\
\text { Becoming more protective } \\
\text { of others (esp. children) } \\
\text { Conditioned fear response } \\
\text { triggered by hearing about } \\
\text { similar events or being in } \\
\text { similar environments }\end{array}$ & $\begin{array}{l}\text { Avoiding thinking and/or } \\
\text { talking about the event } \\
\text { Social isolation } \\
\text { Substance abuse } \\
\text { Self-harm } \\
\text { Self-blame } \\
\text { Bottling up feelings }\end{array}$ & $\begin{array}{l}\text { Taking a positive outlook } \\
\text { Participation in victim or } \\
\text { peer support groups } \\
\text { Medication for physical } \\
\text { and mental health issues } \\
\text { Seeking support from } \\
\text { health professionals } \\
\text { Participation in counselling } \\
\text { or therapy } \\
\text { Trying to challenge } \\
\text { fears/phobias } \\
\text { Realising event is rare and } \\
\text { unlikely to happen again } \\
\text { Advocating for others } \\
\text { affected } \\
\text { Talking with loved ones to } \\
\text { cope with emotional } \\
\text { impact of the event } \\
\text { Learning to accept the } \\
\text { event happened } \\
\text { Learning not to blame } \\
\text { oneself } \\
\text { Turning to religion/faith }\end{array}$ & $\begin{array}{l}\text { Recovery as not having } \\
\text { intrusive flashbacks } \\
\text { Recovery as being able to } \\
\text { cope with lasting pain } \\
\text { Recovery as not letting the } \\
\text { event (or memories) direct } \\
\text { future decisions } \\
\text { Grief subsiding over time } \\
\text { Lessening of impact of } \\
\text { symptoms over time } \\
\text { Being able to move on } \\
\text { from the pain and return } \\
\text { to normal activities } \\
\text { Being able to remember } \\
\text { loved ones fondly without } \\
\text { feeling overwhelmed } \\
\text { Returning to pre-trauma } \\
\text { self is impossible } \\
\text { Emotional pain and } \\
\text { physical scars always } \\
\text { present } \\
\text { Memory of the event } \\
\text { remains / unable to forget } \\
\text { Feeling safe to return to } \\
\text { similar situations and } \\
\text { normal activities }\end{array}$ & $\begin{array}{l}\text { Awareness of fragility of } \\
\text { life } \\
\text { More sensitive towards } \\
\text { and empathetic of others } \\
\text { Personal strength } \\
\text { Not taking life for granted } \\
\text { / living life to the full } \\
\text { Feeling appreciative and } \\
\text { grateful for life }\end{array}$ \\
\hline
\end{tabular}

